\section{Satisfação dos usuários do \\ sistema de saúde brasileiro: \\ fatores associados e diferenças \\ regionais}

\section{User satisfaction in the Brazilian health system: associated factors and regional differences}

Giselle Campozana Gouveia'

Wayner Vieira de Souza'

Carlos F. Luna'

Paulo Roberto Borges de Souza-Júnior"

Célia Landmann Szwarcwald"

' Departamento de Saúde Coletiva do Centro de Pesquisas Aggeu Magalhães (CPqAM).

" Departamento de Informações em Saúde do Instituto de Comunicação e Informação Científica e Tecnológica em Saúde (ICICT) da Fundação Oswaldo Cruz.

Correspondência: Giselle Campozana Gouveia. Instituto de Pesquisas Aggeu Magalhães (CPqAM) - Campus da UFPE, Av. Moraes Rego, s/nº - Cidade Universitária - Recife, PE CEP 50670-420. E-mail: giselle@cpqam.fiocruz.br

\section{Resumo}

Desenvolver modelos de avaliação de satisfação que permitam comparar sistemas de saúde de diversos países é um esforço recente no Brasil. Esse tipo de avaliação representa importante ferramenta para desenvolver estratégias de gestão para o setor. Objetivo: identificar fatores e avaliar diferenças regionais do grau de satisfação dos usuários do sistema de saúde brasileiro, analisando os resultados da Pesquisa Mundial de Saúde (PMS) e Pesquisa Mundial de Saúde com foco na Atenção Básica (PMS-AB). Foi realizado estudo avaliativo de corte transversal, onde as amostras foram selecionadas de forma aleatória. Foram incluídas pessoas maiores de 18 anos que utilizaram o sistema de saúde em períodos anteriores às coletas de dados. Enquadraram-se nos critérios de inclusão 3.932 usuários da PMS e 591 da PMS-AB. Aplicou-se modelo logístico multivariado, tendo como variável dependente a satisfação e, como variáveis independentes, sexo, idade, escolaridade, forma de pagamento de serviço, situação conjugal, autopercepção de saúde, doença de longa duração, diagnóstico de depressão, tristeza, número de moradores por domicílio, tipo de construção, estratos geográficos e indicador de bens. Os resultados revelaram que ser jovem, usuário exclusivo do SUS, ter baixa escolaridade e autopercepção de saúde ruim gerou mais chances de insatisfação dos usuários com o sistema de saúde brasileiro. Os moradores da Região Sul do país estão mais satisfeitos com o atendimento em saúde, enquanto para pernambucanos as chances de satisfação são menores quando se avalia a qualidade dos serviços de saúde. Os moradores desse estado apresentaram maior insatisfação na resolução de seus problemas de saúde quando precisaram de internação.

Palavras-chave: Diferenças regionais. Sistema de saúde. Satisfação de usuários. Modelos de avaliação. 


\section{Abstract}

Developing evaluation models of satisfaction that allow comparing health systems in different countries is a recent effort in Brazil. This is an important tool for building management strategies for the health sector. Objective: To evaluate regional variations related to the degree of user satisfaction with the Brazilian healthcare system, analyzing the results of the World Health Survey (WHS) and World Health Survey Focusing on Primary Health Care (WHS-PHC). The evaluation was done, based on a crosssectional study with a random sample. People over 18 years of age who used the health system in periods before data collection were included. There were 3,932 users of WHS and 591 of WHS-PHC that fit these criteria. A multivariate logistic model was applied using satisfaction as the dependent variable and as independent variables: sex, age, educational level, service payment type, marital status, self-perception of health, long-term illness, diagnosis of depression, sadness, number of residents per household, type of house building, geographical stratum, and household goods index. The results revealed that being young and an exclusive user of the public National Health System, having low schooling and poor selfperception of health increased the chances of lower user satisfaction. Residents of the Southern Region of the country were more satisfied with their healthcare, while in Pernambuco, chances of satisfaction were lower when evaluating the quality of health services. The residents of this state showed greater satisfaction in solving their health problems when inpatient care was needed.

Keywords: Regional variations. Health system. User satisfaction. Evaluation models.

\section{Introdução}

Atualmente, diversas iniciativas voltadas para avaliação em saúde vêm sendo desenvolvidas no Brasil, podendo-se afirmar que as mesmas não se restringem à academia, bem como permitem uma enorme multiplicidade de recortes do real e abordagens metodológicas diferentes, devido à sua natureza e abrangência.

O Ministério da Saúde tem encomendado vários estudos nessa perspectiva, não só pela exigência de financiadores externos, mas devido a uma crescente consciência de responsabilização entre seus técnicos e dirigentes ${ }^{1}$.

Como iniciativa da Organização Mundial de Saúde (OMS), entre os anos de 2003 e 2005 foram realizadas no Brasil duas pesquisas, tendo como objetivo principal avaliar o desempenho do Sistema de Saúde brasileiro. A execução destas pesquisas significou a oportunidade de desenvolver instrumental e de suprir de informações o sistema nacional de saúde. No ano de 2003, essa pesquisa foi realizada em todo território nacional, sendo chamada de Pesquisa Mundial de Saúde (PMS), e em 2005 foi realizada uma nova pesquisa com amostra representativa em dois Estados brasileiros, Rio de Janeiro e Pernambuco, conhecida como Pesquisa Mundial de Saúde com foco na Atenção Básica (PMS-AB).

Os dados gerados por essas duas pesquisas ainda estão sendo analisados e têm permitido, entre outras coisas, gerar informações acerca da resposta do sistema de saúde frente às demandas dos usuários.

A abordagem sistêmica concebida por Donabedian², baseada na avaliação dos resultados, coloca que estes estão relacionados com o produto das ações em saúde e com a modificação no estado de saúde de indivíduos e da população. Mais especificamente falando, avaliamse as características relacionais entre os agentes das ações, usuário e profissional de saúde, onde se analisa a percepção do usuário sobre as práticas de saúde, ou seja, a satisfação dos usuários. 
Com a promoção do controle social, da prática de avaliação dos serviços por meio de perguntas aos usuários e o fortalecimento da participação da comunidade nos processos de planejamento e avaliação, os estudos de satisfação passaram a ter um importante papel no cenário brasileiro ${ }^{3}$.

Alguns autores ${ }^{4,5}$ destacam que esses tipos de estudos trazem no seu bojo a avaliação da qualidade da assistência prestada aos indivíduos através da análise da qualidade da atenção. Para Murray; Kawabata e Valentine $^{6}$, os inquéritos de satisfação não refletem necessariamente as variações na forma como as pessoas estão realmente sendo tratadas pelo sistema, o que reforça a tese de que a percepção dos cuidados é influenciada pela subjetividade do conceito de qualidade e pela expectativa do usuário, o que em muitas situações dificulta este tipo de avaliação. Segundo Esperidião e $\operatorname{Trad}^{3}$, a satisfação envolve algumas dimensões ligadas aos aspectos dos serviços, tais como acesso, qualidade, estrutura física e organizacional, aspectos inerentes à relação médico-paciente etc.

A importância do esforço para desenvolver modelos de avaliação de satisfação, a fim de permitir a comparação de resultados entre sistemas de saúde de diversos países, e dentro do país, é recente no Brasil. Neste caso, um país com desigualdades socioeconômicas importantes, que se distribuem diferentemente de acordo com o lugar, a escolaridade, a raça ou cor da pele das pessoas, por exemplo ${ }^{7}$. Segundo a $\mathrm{OPAS}^{8}$, o Brasil não é um país pobre, mas sim extremamente injusto e desigual, no qual um grande número de pessoas continua enfrentando enormes dificuldades para exercer a cidadania.

Historicamente e na atualidade o país enfrenta problemas estruturais, inclusive no setor saúde, e esse tipo de avaliação pode representar uma importante ferramenta para o desenvolvimento de estratégias de gestão para o setor.

O sistema de saúde brasileiro é fragmentado, e seus diferentes pontos de atenção à saúde estão muito concentrados e não se comunicam adequadamente, o que tem gerado ineficiência e custos sociais para a população ${ }^{9}$. O monitoramento das desigualdades em saúde, em diferentes dimensões que não exclusivamente a geográfica, se configura como essencial para o processo de evolução do sistema de saúde brasileiro.

Segundo Szwarcwald et al. ${ }^{10}$, evidências apontam para um forte gradiente social no âmbito da saúde da população, invariavelmente desfavorável para grupos sociais menos privilegiados. A região das Américas é, entre todas no mundo, a que apresenta maiores desigualdades internas do ponto de vista de situação socioeconômica de seus grupos populacionais e de condições materiais de vida em geral, o que se reflete em grandes desigualdades, tanto no estado de saúde de grupos populacionais, como no acesso e utilização de serviços de atenção à saúde ${ }^{7}$.

A Organização Pan-Americana de Saúde $(\mathrm{OPAS})^{11}$ aponta nos anos recentes para o crescimento da economia brasileira, o que elevou a renda média em todos os estratos da população, mas a distribuição desigual fez aumentar as diferenças internas preexistentes.

Análises baseadas na aplicação do Índice de Desenvolvimento Humano (IDH), que são usadas como uma maneira padronizada de avaliação e medida do bem-estar de uma população, revelaram que o Brasil está muito próximo do limite a partir do qual os países são incluídos no grupo de nível mais elevado (entre 0,800 e 1). Entretanto, análises de dados realizadas em 2005 revelaram disparidades internas significativas, em que o IDH para o Brasil foi de 0,794 , sendo que as diferenças entre as regiões geográficas variou de 0,720 para a região Nordeste até 0,829 para a região $\mathrm{Sul}^{12}$.

As desigualdades regionais que caracterizam a sociedade brasileira têm origem antiga. Segundo Negrii ${ }^{13}$, em 1986 a partição dos recursos do Instituto Nacional de Assistência Médica da Previdência Social demonstrava essa diferença, pois $59 \%$ dos recursos destinados à saúde iam para o Sudeste ( $44 \%$ da população do país), enquanto 
apenas $20 \%$ eram destinados às Regiões Norte e Nordeste (34\% da população). Essa distribuição não equânime dos recursos acabou aumentando as desigualdades no setor.

Muitos esforços têm sido empregados pelos gestores do sistema de saúde que buscam a melhoria do atendimento em saúde no país com vistas à equidade do sistema, prevista na Constituição Federal de 1988. Entretanto, alguns autores ${ }^{14}$, baseados nos resultados da Pesquisa Nacional por Amostra de Domicílios de 1998 e 2003, demonstraram que não houve alterações nas desigualdades sociais em saúde neste período.

Alguns estudos ${ }^{10}$ têm enfatizado a forte associação entre condições de saúde e o nível socioeconômico das populações, e estes têm sido considerados como importantes componentes de avaliação de sistemas de saúde. Contudo, existe uma escassez de estudos nacionais sobre o tema, explicada parcialmente pela limitação de dados disponíveis em nível nacional.

Baseado nos pressupostos acima e com o riquíssimo material disponível em nível nacional, este artigo buscou avaliar os fatores associados à satisfação, bem como as diferenças regionais do grau de satisfação dos usuários do sistema de saúde brasileiro, analisando os resultados das duas pesquisas: PMS brasileira e PMS-AB realizada no Estado de Pernambuco.

Os resultados dessas pesquisas passaram por diversas análises e neste artigo procura-se apresentar uma parte destes.

\section{Métodos}

Estudo avaliativo de corte transversal realizado no ano de 2003 para todo o território nacional e em 2005 para o Estado de Pernambuco.

Foram incluídos neste estudo todos os indivíduos com idade igual ou acima de 18 anos que se internaram nos cinco anos anteriores ao período da coleta de dados da PMS brasileira, todos os indivíduos que utilizaram serviços ambulatoriais de saúde no ano anterior à referida coleta, e todos os indivíduos que receberam atendimento ambulatorial ou internação no ano anterior à coleta de dados da PMS-AB realizada no Estado de Pernambuco.

As amostras para as duas pesquisas foram selecionadas de forma aleatória. $\mathrm{Na}$ PMS brasileira, foram incluídos 5.000 usuários residentes em 250 setores censitários de todo país ${ }^{15}$, sendo que desses apenas 3.932 se enquadravam nos critérios de inclusão deste trabalho. Da mesma forma, a amostra da PMS-AB realizada em Pernambuco incluiu 720 usuários em todo Estado, mas apenas 591 se enquadravam nos critérios.

Foram utilizados os bancos de dados da PMS brasileira e da PMS-AB de Pernambuco, cedidos oficialmente pela coordenação nacional da Pesquisa Mundial de Saúde no Brasil. Essa pesquisa foi aprovada pelo Comitê de Ética do Centro de Pesquisas Aggeu Magalhães. Os dois bancos de dados foram trabalhados separadamente.

Para a análise descritiva do perfil socioeconômico e demográfico da população foram utilizadas as seguintes variáveis: sexo, faixa etária (18 a 39 anos e 40 anos e mais), escolaridade ( $1^{\circ}$ grau incompleto e $1^{\circ}$ grau completo e mais), situação de trabalho (empregados e não-empregados - incluindo pessoas não economicamente ativas) e número de bens no domicílio (até 1 bem e mais de 1 bem).

A investigação abrangeu um conjunto de 16 perguntas que expressavam o grau de satisfação do usuário, por meio de cinco níveis de resposta (do nível 1 - muito bom, ao nível 5 - muito ruim), com os seguintes aspectos: tempo gasto com o deslocamento até o serviço; tempo de espera para o atendimento; tratamento respeitoso por parte dos profissionais de saúde; respeito durante exames físicos; clareza nas explicações dos profissionais; disponibilidade de tempo para fazer perguntas sobre o problema de saúde ou tratamento; possibilidade de obter informações sobre tratamentos ou exames alternativos; participação na tomada de decisões; privacidade junto aos profissionais de saúde; sigilo das informações; liberdade 
de escolha do profissional de saúde; limpeza das instalações dos serviços e espaço disponível das salas de espera e atendimento, além de três variáveis dicotômicas referentes às habilidades do profissional de saúde; disponibilidade de equipamentos e disponibilidade de medicamentos.

Construiu-se uma matriz com as 16 perguntas do estudo (variáveis) x indivíduos e, com o emprego da Análise de Componentes Principais (ACP), procedeu-se à extração dos componentes principais (eixos). Visando separar as variáveis, a ACP produziu a matriz de cargas "factors loadings", que representam as correlações de cada variável com cada eixo encontrado. Após este processo foram gerados os "factors scores", que passaram a representar o grau de satisfação de cada indivíduo em relação a cada eixo encontrado.

Os escores de cada componente principal foram transformados em uma escala que variava de 0 a 100, em que o valor zero correspondia ao escore mínimo de satisfação e o valor 100 ao nível máximo. Os indivíduos classificados como satisfeitos foram aqueles que tiveram escore superior ao valor mediano dos escores de satisfação. Foram realizadas análises baseadas nos componentes principais encontrados e no tipo de assistência recebida (ambulatorial e internação hospitalar).

Foi aplicado um modelo logístico multivariado que tinha como variável dependente a satisfação (escore de satisfação dicotomizado) e como variáveis independentes aquelas relacionadas aos indivíduos (sexo, idade, escolaridade, forma de pagamento de serviço de saúde, situação conjugal, autopercepção de saúde, doença de longa duração, diagnóstico de depressão, tristeza nos últimos 12 meses) e aquelas relacionadas às características do domicílio (número de moradores por domicílio, tipo de construção, estratos geográficos e indicador de bens). A força de associação entre as variáveis independentes e a dependente foi expressa pela Odds Ratio (OR), e respectivo intervalo de confiança de $95 \%$. Foi considerado como fator de seleção para a variável entrar no modelo logístico multivariado a mesma ter significância menor que $20 \%$, ou seja, $p<$ 0,2 na análise univariada.

Para efeito de comparação, os dados da PMS brasileira foram agregados segundo os seguintes estratos geográficos: usuários do sistema de saúde de Pernambuco; usuários do sistema de saúde da Região Nordeste, excluindo-se os usuários do Estado de Pernambuco; usuários do sistema de saúde da Região Norte; usuários do sistema de saúde da Região Centro-Oeste; usuários do sistema de saúde da Região Sudeste; usuários do sistema de saúde da Região Sul. Para as comparações regionais, tomou-se como referência a Região Sudeste.

Para a PMS brasileira, a partir da variável número de bens do domicílio foi construído um índice chamado indicador de bens (IB), conforme metodologia empregada por Szwarcwald et al. ${ }^{16}$. Utilizou-se a fórmula IB $=\Sigma_{i}\left(1-f_{i}\right) \mathrm{b}_{i}$, em que $i$ varia de 1 até 10 bens, $\mathrm{b}_{i}$ é igual a 1 ou a zero, respectivamente na presença ou ausência de bem. O peso atribuído à presença de cada bem no domicílio foi o complementar da freqüência relativa $\left(f_{i}\right)$ de cada bem na amostra total, isto é, quanto mais rara for a presença do bem, maior o peso atribuído ao mesmo.

Em seguida foram selecionadas e recodificadas as variáveis do estudo, de forma a deixá-las dicotômicas. As variáveis "indicador de bens e número de moradores por domicílio" permaneceram em suas escalas originais nas análises. $\mathrm{O}$ método utilizado para a seleção das variáveis na regressão logística foi o Backward e para a análise estatística foi utilizado o programa SPSS (versão 8.0).

\section{Resultados}

A análise do perfil socioeconômico e demográfico dos usuários entrevistados na PMS brasileira revelou que $31 \%$ das pessoas que receberam algum tipo de atendimento ( $\mathrm{n}$ = 3932), relataram assistência de saúde com internação nos cinco anos anteriores à pesquisa. Desses, $71,0 \%$ foram atendidos pelo SUS. Dos demais participantes que tiveram pelo menos um atendimento ambu- 
latorial no ano que antecedeu à entrevista, $60 \%$ utilizaram o SUS.

Na PMS brasileira não foram observadas diferenças entre o sexo, a idade e a quantidade de bens das pessoas que tiveram atendimento ambulatorial, independentemente da forma de pagamento da assistência. A análise revelou que, para os usuários do SUS, houve predominância da baixa escolaridade (66\%) e de não-empregados (51\%), enquanto para os indivíduos que pagaram pelo atendimento predominaram pessoas com alta escolaridade (70\%) e empregados (59\%).

Em relação ao perfil das pessoas que se internaram pelo SUS, houve predominância de jovens (59\%), mulheres (68\%), baixa escolaridade (68\%), não-empregados (60\%) e com mais bens no domicílio (91\%). Das que pagaram pela internação predominaram pessoas com 40 anos ou mais (52\%), do sexo feminino (64\%), com alta escolaridade (65\%) e, da mesma forma que os usuários do SUS, houve predomínio dos não-empregados (52\%) e com mais bens por domicílio (99\%).

Dos 591 usuários entrevistados na PMS-AB de Pernambuco, 87,6\% receberam assistência ambulatorial no ano anterior à pesquisa, sendo 73,0\% usuários do SUS. Em relação aos usuários que se internaram no ano anterior à pesquisa, 84,9\% utilizaram o SUS no seu atendimento.

Dos usuários que tiveram atendimento ambulatorial na PMS-AB, aproximadamente $60 \%$ tinham entre 18 e 39 anos de idade, com predominância do sexo feminino. Pessoas com ensino fundamental completo representavam $69,3 \%$ dos usuários Não-SUS, enquanto para os usuários do SUS predominaram aqueles com ensino fundamental incompleto (70,6\%). Mais de $50 \%$ dos usuários, independentemente da forma de pagamento, são pessoas que não trabalham e com pouco número de bens, sendo que para o SUS esses percentuais são superiores aos encontrados para os usuários Não-SUS.

Das pessoas que relataram internação, a análise revelou que a faixa etária de 18 a
39 anos foi predominante tanto entre os usuários do SUS $(67,8 \%)$ como entre os usuários Não-SUS (72,8\%). O sexo feminino foi predominante, independentemente da forma de pagamento da assistência (58,3\% Não-SUS e 78,7\% SUS). A grande maioria dos usuários Não-SUS tem ensino fundamental completo ou mais ( $83,3 \%$ ), enquanto entre os usuários do SUS o predomínio foi de pessoas com o nível de escolaridade do ensino fundamental incompleto $(63,9 \%)$. Como revelado para o atendimento ambulatorial, mais de $50 \%$ dos usuários, independentemente da forma de pagamento, são pessoas que não trabalham e com pouco número de bens, sendo que para o SUS esses percentuais são bem superiores aos encontrados para os usuários Não-SUS.

A ACP identificou três eixos sendo os mesmos relativos à qualidade dos profissionais de saúde, qualidade dos serviços de saúde e resolutividade dos problemas de saúde, resultado este compatível como o encontrado por GOUVEIA et al. ${ }^{17}$.

As Tabelas 1, 2 e 3 apresentam os resultados da análise univariada das duas pesquisas para os três eixos e pelo tipo de atendimento recebido. Destacam-se em negrito as variáveis que não foram selecionadas para entrar no modelo final da regressão logística, pois apresentaram o valor de $p>20 \%$ na análise univariada ou na análise multivariada.

A análise dos resultados do modelo final da regressão logística, demonstrados na Tabela 4 , revelou que entre os usuários que se dizem satisfeitos com a qualidade dos profissionais de saúde durante o atendimento ambulatorial na PMS brasileira, as mulheres apresentam $30 \%$ mais chances de estarem mais satisfeitas do que os homens, enquanto as pessoas de baixa escolaridade, usuárias do SUS, com auto-percepção de saúde ruim, e com maior número de pessoas residindo no mesmo domicílio, apresentaram menor chance de estarem satisfeitas com o atendimento no sistema de saúde.

Quanto à análise dos dados da PMS-AB realizada em Pernambuco, a única variável que mostrou estar associada à satisfação 
Tabela 1- Análise univariada da associação das características individuais e domiciliares de indivíduos que receberam atendimento ambulatorial e internação hospitalar e que se dizem satisfeitos em relação à qualidade dos profissionais de saúde. Brasil, 2003 e Pernambuco 2005.

Table 1 - Univariate analysis of association of individual and household characteristics of individuals who received outpatient care and hospitalization to the degree of satisfaction with the quality of healthcare workers. Brazil, 2003 and Pernambuco, 2005.

\begin{tabular}{|c|c|c|c|c|c|c|c|c|c|c|c|c|}
\hline \multirow[t]{3}{*}{ Características Gerais } & \multicolumn{6}{|c|}{ Atendimento ambulatorial } & \multicolumn{6}{|c|}{ Internação hospitalar } \\
\hline & \multicolumn{3}{|c|}{ PMS brasileira } & \multicolumn{3}{|c|}{ PMS_AB de Pernambuco } & \multicolumn{3}{|c|}{ PMS brasileira } & \multicolumn{3}{|c|}{ PMS_AB de Pernambuco } \\
\hline & OR & $95 \% \mathrm{Cl}$ & $\mathrm{p}$-value & OR & $95 \% \mathrm{Cl}$ & $\mathrm{p}$-value & OR & $95 \% \mathrm{Cl}$ & $\mathrm{p}$-value & OR & $95 \% \mathrm{Cl}$ & $\mathrm{p}$-value \\
\hline \multicolumn{13}{|l|}{ Sexo } \\
\hline Masculino & 1,0 & - & - & 1,0 & - & - & 1,0 & - & - & 1,0 & - & - \\
\hline Feminino & 1,2 & $1,0-1,5$ & 0,026 & 1,2 & $0,8-1,7$ & 0,452 & 1,0 & $0,8-1,3$ & 0,733 & 2,0 & $0,6-6,4$ & 0,251 \\
\hline \multicolumn{13}{|l|}{ Faixa etária } \\
\hline 40 anos e + & 1,0 & - & - & 1,0 & - & - & 1,0 & - & - & 1,0 & - & - \\
\hline 18 a 39 anos & 1,1 & $0,9-1,3$ & 0,377 & 1,4 & $0,9-2,1$ & 0,090 & 0,7 & $0,5-0,8$ & $<0,001$ & 2,0 & $0,7-5,6$ & 0,183 \\
\hline \multicolumn{13}{|l|}{ Escolaridade } \\
\hline $1^{\circ}$ grau completo e + & 1,0 & - & - & 1,0 & - & - & 1,0 & - & - & 1,0 & - & - \\
\hline $1^{\circ} \mathrm{grau}$ incompleto & 0,5 & $0,4-0,6$ & $<0,001$ & 0,5 & $0,4-0,8$ & 0,002 & 0,7 & $0,5-0,8$ & 0,001 & 0,2 & $0,1-0,6$ & 0,005 \\
\hline \multicolumn{13}{|l|}{ Forma de Pagamento } \\
\hline Não SUS & 1,0 & - & - & 1,0 & - & - & 1,0 & - & - & 1,0 & - & - \\
\hline$\underline{\text { SUS }}$ & 0,3 & $0,3-0,4$ & $<0,001$ & 0,5 & $0,3-0,8$ & 0,007 & 0,3 & $0,2-0,4$ & $<0,001$ & 0,4 & $0,1-1,7$ & 0,229 \\
\hline \multicolumn{13}{|l|}{ Estado conjugal } \\
\hline Solteiro (a) & 1,0 & - & - & 1,0 & - & - & 1,0 & - & - & 1,0 & - & - \\
\hline Casado (a) & 0,8 & $0,7-1,0$ & 0,058 & 0,7 & $0,5-1,2$ & 0,172 & 1,2 & $0,9-1,6$ & 0,282 & 0,6 & $0,1-2,3$ & 0,440 \\
\hline Separado (a) & 0,9 & $0,6-1,3$ & 0,480 & 1,1 & $0,5-2,4$ & 0,718 & 1,1 & $0,7-1,8$ & 0,771 & 0,3 & $0,0-2,1$ & 0,204 \\
\hline Viúvo (a) & 1,0 & $0,6-1,4$ & 0,833 & 0,5 & $0,2-1,1$ & 0,076 & 1,0 & $0,6-1,7$ & 0,983 & 0,3 & $0,0-2,4$ & 0,239 \\
\hline \multicolumn{13}{|l|}{$\begin{array}{l}\text { Auto-percepção de } \\
\text { saúde }\end{array}$} \\
\hline Boa & 1,0 & - & - & 1,0 & - & - & 1,0 & - & - & 1,0 & - & - \\
\hline Ruim & 0,5 & $0,4-0,6$ & $<0,001$ & 0,9 & $0,6-1,3$ & 0,525 & 0,6 & $0,5-0,8$ & $<0,001$ & 1,1 & $0,4-2,9$ & 0,809 \\
\hline \multicolumn{13}{|l|}{$\begin{array}{l}\text { Doença de longa } \\
\text { duração }\end{array}$} \\
\hline Não & 1,0 & - & - & 1,0 & - & - & 1,0 & - & - & 1,0 & - & - \\
\hline Sim & 0,7 & $0,6-0,9$ & 0,003 & 0,9 & $0,5-1,4$ & 0,532 & & $0,9-1,4$ & 0,430 & 0,5 & $0,2-1,6$ & 0,251 \\
\hline \multicolumn{13}{|l|}{$\begin{array}{l}\text { Diagnóstico de } \\
\text { depressão }\end{array}$} \\
\hline Não & 1,0 & - & - & 1,0 & - & - & 1,0 & - & - & 1,0 & - & - \\
\hline$\underline{\text { Sim }}$ & 1,3 & $1,0-1,6$ & 0,048 & 1,0 & $0,6-1,9$ & 0,890 & 1,0 & $0,8-1,3$ & 0,789 & 0,7 & $0,2-2,2$ & 0,487 \\
\hline \multicolumn{13}{|l|}{$\begin{array}{l}\text { Tristeza nos últimos } 12 \\
\text { meses }\end{array}$} \\
\hline Não & 1,0 & - & - & 1,0 & - & - & 1,0 & - & - & 1,0 & - & - \\
\hline Sim & 0,9 & $0,7-1,0$ & 0,089 & 0,7 & $0,5-1,1$ & 0,103 & 0,8 & $0,6-1,0$ & 0,019 & 1,0 & $0,4-2,7$ & 0,926 \\
\hline \multicolumn{13}{|l|}{ Tipo de construção } \\
\hline Material permanente & 1,0 & - & - & 1,0 & - & - & 1,0 & - & - & 1,0 & - & - \\
\hline Material não permanente & 0,5 & $0,3-0,8$ & 0,012 & 1,3 & $0,4-4,1$ & 0,648 & 0,6 & $0,4-1,0$ & 0,072 & 0,0 & - & - \\
\hline \multicolumn{13}{|l|}{ Estrato geográfico } \\
\hline Sudeste & 1,0 & - & - & - & - & - & 1,0 & - & - & - & - & - \\
\hline Norte & 1,2 & $0,7-2,1$ & 0,485 & - & - & - & 0,7 & $0,4-1,2$ & 0,193 & - & - & - \\
\hline $\begin{array}{l}\text { Nordeste sem } \\
\text { Pernambuco }\end{array}$ & 0,6 & $0,5-0,8$ & $<0,001$ & - & - & - & 0,6 & $0,5-0,9$ & 0,003 & - & - & - \\
\hline Sul & 1,6 & $1,3-2,1$ & $<0,001$ & - & - & - & 1,6 & $1,2-2,2$ & 0,004 & - & - & - \\
\hline Centro-Oeste & 1,2 & $0,8-1,8$ & 0,282 & - & - & - & 1,6 & $1,0-2,6$ & 0,039 & - & - & - \\
\hline Pernambuco & 0,9 & $0,6-1,4$ & 0,704 & - & - & - & 0,5 & $0,3-0,8$ & 0,007 & - & - & - \\
\hline Indicador de Bens & 1,5 & $1,4-1,6$ & $<0,001$ & 1,2 & $1,0-1,5$ & 0,065 & 1,6 & $1,4-1,8$ & $<0,001$ & 2,2 & $1,2-4,0$ & 0,009 \\
\hline $\begin{array}{l}\text { Moradores por } \\
\text { domicílio }\end{array}$ & 0,9 & $0,9-1,0$ & $<0,001$ & 1,0 & $0,9-1,1$ & 0,578 & 0,9 & $0,9-1,0$ & 0,001 & 1,0 & $0,7-1,3$ & 0,787 \\
\hline
\end{tabular}


Tabela 2 - Análise univariada das características individuais e domiciliares de indivíduos que receberam atendimento ambulatorial e internação hospitalar e que se dizem satisfeitos em relação à qualidade dos serviços de saúde. Brasil, 2003 e Pernambuco 2005.

Table 2 - Univariate analysis of individual and household characteristics of individuals who received outpatient care and hospitalization and who said they were satisfied with the quality of healthcare services. Brazil, 2003 and Pernambuco, 2005.

\begin{tabular}{|c|c|c|c|c|c|c|c|c|c|c|c|c|}
\hline \multirow[t]{3}{*}{ Características Gerais } & \multicolumn{6}{|c|}{ Atendimento ambulatorial } & \multicolumn{6}{|c|}{ Internação hospitalar } \\
\hline & \multicolumn{3}{|c|}{ PMS brasileira } & \multicolumn{3}{|c|}{ PMS_AB de Pernambuco } & \multicolumn{3}{|c|}{ PMS brasileira } & \multicolumn{3}{|c|}{ PMS_AB de Pernambuco } \\
\hline & OR & $95 \% \mathrm{Cl}$ & $p$-value & OR & $95 \% \mathrm{Cl}$ & $\mathrm{p}$-value & OR & $95 \% \mathrm{Cl}$ & $\mathrm{p}$-value & OR & $95 \% \mathrm{Cl}$ & $\mathrm{p}$-value \\
\hline \multicolumn{13}{|l|}{ Sexo } \\
\hline Masculino & 1,0 & - & - & 1,0 & - & - & 1,0 & - & - & 1,0 & - & - \\
\hline Feminino & 0,9 & $0,7-1,0$ & 0,104 & 0,6 & $0,3-1,0$ & 0,050 & 1,0 & $0,8-1,3$ & 0,877 & 0,9 & $0,3-2,9$ & 0,855 \\
\hline \multicolumn{13}{|l|}{ Faixa etária } \\
\hline 40 anos e + & 1,0 & - & - & 1,0 & - & - & 1,0 & - & - & 1,0 & - & - \\
\hline 18 a 39 anos & 0,9 & $0,7-1,0$ & 0,082 & 1,0 & $0,6-1,8$ & 0,967 & 0,8 & $0,6-1,0$ & 0,049 & 0,6 & $0,2-1,8$ & 0,373 \\
\hline \multicolumn{13}{|l|}{ Escolaridade } \\
\hline $1^{\circ}$ grau completo e + & 1,0 & - & - & 1,0 & - & - & 1,0 & - & - & 1,0 & - & - \\
\hline $1^{\circ} \mathrm{grau}$ incompleto & 0,8 & $0,6-0,9$ & 0,003 & 0,5 & $0,3-0,8$ & 0,009 & 0,7 & $0,5-0,8$ & $<0,001$ & 1,0 & $0,4-2,5$ & 0,948 \\
\hline \multicolumn{13}{|l|}{ Forma de Pagamento } \\
\hline Não SUS & 1,0 & - & - & 1,0 & - & - & 1,0 & - & - & 1,0 & - & - \\
\hline$\underline{\text { SUS }}$ & 0,3 & $0,2-0,4$ & $<0,001$ & 0,2 & $0,1-0,3$ & $<0,001$ & 0,3 & $0,2-0,4$ & $<0,001$ & 1,3 & $0,4-4,8$ & 0,658 \\
\hline \multicolumn{13}{|l|}{ Estado conjugal } \\
\hline Solteiro (a) & 1,0 & - & - & 1,0 & - & - & 1,0 & - & - & 1,0 & - & - \\
\hline Casado (a) & 1,0 & $0,8-1,2$ & 0,682 & 0,9 & $0,5-1,5$ & 0,631 & 1,4 & $1,1-1,9$ & 0,019 & 2,6 & $0,6-10,1$ & 0,182 \\
\hline Separado (a) & 0,9 & $0,6-1,3$ & 0,503 & 0,3 & $0,1-1,1$ & 0,066 & 1,0 & $0,6-1,7$ & 0,999 & 1,5 & $0,2-10,1$ & 0,719 \\
\hline Viúvo (a) & 1,1 & $0,7-1,6$ & 0,773 & 0,3 & $0,1-1,5$ & 0,138 & 1,2 & $0,8-2,0$ & 0,407 & 3,7 & $0,4-34,5$ & 0,252 \\
\hline \multicolumn{13}{|l|}{$\begin{array}{l}\text { Auto-percepção de } \\
\text { saúde }\end{array}$} \\
\hline Boa & 1,0 & - & - & 1,0 & - & - & 1,0 & - & - & 1,0 & - & - \\
\hline Ruim & 0,6 & $0,5-0,7$ & $<0,001$ & 0,9 & $0,5-1,5$ & 0,646 & 0,6 & $0,5-0,8$ & $<0,001$ & 1,5 & $0,6-4,0$ & 0,374 \\
\hline \multicolumn{13}{|l|}{$\begin{array}{l}\text { Doença de longa } \\
\text { duração }\end{array}$} \\
\hline Não & 1,0 & - & - & 1,0 & - & - & 1,0 & - & - & 1,0 & - & - \\
\hline$\underline{\text { Sim }}$ & 0,8 & $0,6-1,0$ & 0,018 & 0,9 & $0,5-1,8$ & 0,819 & 1,0 & $0,8-1,3$ & 0,875 & 0,6 & $0,2-2,0$ & 0,432 \\
\hline \multicolumn{13}{|l|}{$\begin{array}{l}\text { Diagnóstico de } \\
\text { depressão }\end{array}$} \\
\hline Não & 1,0 & - & - & 1,0 & - & - & 1,0 & - & - & 1,0 & - & - \\
\hline Sim & 1,1 & $0,9-1,4$ & 0,348 & 0,6 & $0,3-1,4$ & 0,248 & 1,0 & $0,8-1,3$ & 0,842 & 1,8 & $0,5-6,3$ & 0,336 \\
\hline \multicolumn{13}{|l|}{$\begin{array}{l}\text { Tristeza nos últimos } 12 \\
\text { meses }\end{array}$} \\
\hline Não & 1,0 & - & - & 1,0 & - & - & 1,0 & - & - & 1,0 & - & - \\
\hline$\underline{\text { Sim }}$ & 0,8 & $0,7-1,0$ & 0,027 & 0,6 & $0,4-1,1$ & 0,091 & 0,8 & $0,6-1,0$ & 0,016 & 0,3 & $0,1-0,9$ & 0,032 \\
\hline \multicolumn{13}{|l|}{ Tipo de construção } \\
\hline Material permanente & 1,0 & - & - & 1,0 & - & - & 1,0 & - & - & 1,0 & - & - \\
\hline Material não permanente & 0,8 & $0,4-1,3$ & 0,338 & 0,7 & $0,1-5,4$ & 0,759 & 0,7 & $0,4-1,3$ & 0,255 & - & - & - \\
\hline \multicolumn{13}{|l|}{ Estrato geográfico } \\
\hline Sudeste & 1,0 & - & - & - & - & - & 1,0 & - & - & - & - & - \\
\hline Norte & 0,9 & $0,5-1,6$ & 0,745 & - & - & - & 0,9 & $0,5-1,5$ & 0,664 & - & - & - \\
\hline $\begin{array}{l}\text { Nordeste sem } \\
\text { Pernambuco }\end{array}$ & 0,6 & $0,5-0,8$ & $<0,001$ & - & - & - & 0,8 & $0,6-1,0$ & 0,085 & - & - & - \\
\hline Sul & 1,5 & $1,1-1,9$ & 0,006 & - & - & - & 0,8 & $0,6-1,1$ & 0,200 & - & - & - \\
\hline Centro-Oeste & 1,1 & $0,8-1,7$ & 0,472 & - & - & - & 0,9 & $0,6-1,4$ & 0,685 & - & - & - \\
\hline Pernambuco & 0,6 & $0,4-0,9$ & 0,017 & - & - & - & 0,5 & $0,3-0,8$ & 0,008 & - & - & - \\
\hline Indicador de Bens & 1,3 & $1,2-1,4$ & $<0,001$ & 1,4 & $1,1-1,7$ & 0,012 & 1,4 & $1,3-1,5$ & $<0,001$ & 0,9 & $0,5-1,4$ & 0,577 \\
\hline $\begin{array}{l}\text { Moradores por } \\
\text { domicílio }\end{array}$ & 0,9 & $0,9-1,0$ & $<0,001$ & 0,9 & $0,8-1,1$ & 0,363 & 0,9 & $0,9-1,0$ & 0,006 & 0,9 & $0,6-1,2$ & 0,320 \\
\hline
\end{tabular}


Tabela 3 - Análise univariada das características individuais e domiciliares de indivíduos que receberam atendimento ambulatorial e internação hospitalar e que se dizem satisfeitos em relação à resolutividade dos problemas de saúde. Brasil, 2003 e Pernambuco 2005.

Table 3 - Univariate analysis of individual and household characteristics of individuals who received outpatient care and hospitalization and who said they were satisfied with the resolution of health problems. Brazil, 2003 and Pernambuco, 2005.

\begin{tabular}{|c|c|c|c|c|c|c|c|c|c|c|c|c|}
\hline \multirow[t]{3}{*}{ Características Gerais } & \multicolumn{6}{|c|}{ Atendimento ambulatorial } & \multicolumn{6}{|c|}{ Internação hospitalar } \\
\hline & \multicolumn{3}{|c|}{ PMS brasileira } & \multicolumn{3}{|c|}{ PMS_AB de Pernambuco } & \multicolumn{3}{|c|}{ PMS brasileira } & \multicolumn{3}{|c|}{ PMS_AB de Pernambuco } \\
\hline & OR & $95 \% \mathrm{Cl}$ & $p$-value & OR & $95 \% \mathrm{Cl}$ & $\mathrm{p}$-value & OR & $95 \% \mathrm{Cl}$ & $\mathrm{p}$-value & OR & $95 \% \mathrm{Cl}$ & $\mathrm{p}$-value \\
\hline \multicolumn{13}{|l|}{ Sexo } \\
\hline Masculino & 1,0 & - & - & 1,0 & - & - & 1,0 & - & - & 1,0 & - & - \\
\hline Feminino & 0,8 & $0,6-0,9$ & 0,007 & 0,9 & $0,6-1,3$ & 0,481 & 1,0 & $0,8-1,3$ & 0,692 & 0,9 & $0,3-3,5$ & 0,920 \\
\hline \multicolumn{13}{|l|}{ Faixa etária } \\
\hline 40 anos e + & 1,0 & - & - & 1,0 & - & - & 1,0 & - & - & 1,0 & - & - \\
\hline 18 a 39 anos & 0,8 & $0,7-1,0$ & 0,023 & 1,5 & $1,0-2,2$ & 0,068 & 1,0 & $0,8-1,3$ & 0,764 & 0,2 & $0,1-0,7$ & 0,010 \\
\hline \multicolumn{13}{|l|}{ Escolaridade } \\
\hline $1^{\circ}$ grau completo e + & 1,0 & - & - & 1,0 & - & - & 1,0 & - & - & 1,0 & - & - \\
\hline $1^{\circ} \mathrm{grau}$ incompleto & 1,7 & $1,4-2,0$ & $<0,001$ & 0,3 & $0,2-0,5$ & $<0,001$ & 1,7 & $1,3-2,1$ & $<0,001$ & 1,3 & $0,4-4,0$ & 0,599 \\
\hline \multicolumn{13}{|l|}{ Forma de Pagamento } \\
\hline Não SUS & 1,0 & - & - & 1,0 & - & - & 1,0 & - & - & 1,0 & - & - \\
\hline SUS & 1,6 & $1,3-1,9$ & $<0,001$ & 0,7 & $0,4-1,1$ & 0,108 & 1,9 & $1,5-2,4$ & $<0,001$ & 0,7 & $0,2-2,8$ & 0,596 \\
\hline \multicolumn{13}{|l|}{ Estado conjugal } \\
\hline Solteiro (a) & 1,0 & - & - & 1,0 & - & - & 1,0 & - & - & 1,0 & - & - \\
\hline Casado (a) & 1,1 & $0,9-1,3$ & 0,365 & 0,8 & $0,5-1,3$ & 0,352 & 1,0 & $0,7-1,3$ & 0,933 & 1,6 & $0,3-8,6$ & 0,611 \\
\hline Separado (a) & 1,1 & $0,8-1,7$ & 0,544 & 0,6 & $0,3-1,3$ & 0,207 & 1,4 & $0,9-2,4$ & 0,169 & 3,5 & $0,4-32,2$ & 0,278 \\
\hline Viúvo (a) & 1,0 & $0,7-1,5$ & 0,900 & 0,7 & $0,3-1,7$ & 0,433 & 0,8 & $0,5-1,3$ & 0,312 & 2,9 & $0,3-32,0$ & 0,388 \\
\hline \multicolumn{13}{|l|}{$\begin{array}{l}\text { Auto-percepção de } \\
\text { saúde }\end{array}$} \\
\hline Boa & 1,0 & - & - & 1,0 & - & - & 1,0 & - & - & 1,0 & - & - \\
\hline Ruim & 1,2 & $1,0-1,4$ & 0,040 & 0,7 & $0,5-1,0$ & 0,043 & 1,5 & $1,2-1,8$ & 0,001 & 1,0 & $0,3-3,0$ & 0,959 \\
\hline \multicolumn{13}{|l|}{$\begin{array}{l}\text { Doença de longa } \\
\text { duração }\end{array}$} \\
\hline Não & 1,0 & - & - & 1,0 & - & - & 1,0 & - & - & 1,0 & - & - \\
\hline Sim & 1,1 & $0,9-1,4$ & 0,224 & 0,9 & $0,5-1,4$ & 0,580 & 1,0 & $0,8-1,3$ & 0,868 & 0,6 & $0,2-2,6$ & 0,535 \\
\hline \multicolumn{13}{|l|}{$\begin{array}{l}\text { Diagnóstico de } \\
\text { depressão }\end{array}$} \\
\hline Não & 1,0 & - & - & 1,0 & - & - & 1,0 & - & - & 1,0 & - & - \\
\hline Sim & 0,8 & $0,6-1,0$ & 0,064 & 0,7 & $0,4-1,3$ & 0,273 & 1,0 & $0,8-1,3$ & 0,850 & 1,8 & $0,5-6,3$ & 0,391 \\
\hline \multicolumn{13}{|l|}{$\begin{array}{l}\text { Tristeza nos últimos } 12 \\
\text { meses }\end{array}$} \\
\hline Não & 1,0 & - & - & 1,0 & - & - & 1,0 & - & - & 1,0 & - & - \\
\hline Sim & 0,9 & $0,7-1,0$ & 0,102 & 0,7 & $0,5-1,0$ & 0,050 & 1,2 & $1,0-1,5$ & 0,109 & 1,3 & $0,4-3,9$ & 0,650 \\
\hline \multicolumn{13}{|l|}{ Tipo de construção } \\
\hline Material permanente & 1,0 & - & - & 1,0 & - & - & 1,0 & - & - & - & - & - \\
\hline Material não permanente & 1,1 & $0,6-1,9$ & 0,792 & 0,1 & $0,0-0,7$ & 0,016 & 1,4 & $0,8-2,3$ & 0,243 & - & - & - \\
\hline \multicolumn{13}{|l|}{ Estrato geográfico } \\
\hline Sudeste & 1,0 & - & - & - & - & - & 1,0 & - & - & - & - & - \\
\hline Norte & 0,4 & $0,2-0,7$ & 0,001 & - & - & - & 1,0 & $0,6-1,8$ & 0,940 & - & - & - \\
\hline $\begin{array}{l}\text { Nordeste sem } \\
\text { Pernambuco }\end{array}$ & 1,4 & $1,1-1,8$ & 0,002 & - & - & - & 1,3 & $1,0-1,8$ & 0,049 & - & - & - \\
\hline Sul & 0,7 & $0,6-1,0$ & 0,030 & - & - & - & 0,9 & $0,7-1,2$ & 0,552 & - & - & - \\
\hline Centro-Oeste & 1,1 & $0,7-1,5$ & 0,727 & - & - & - & 0,6 & $0,4-1,0$ & 0,060 & - & - & - \\
\hline Pernambuco & 1,5 & $0,9-2,3$ & 0,094 & - & - & - & 0,5 & $0,3-0,8$ & 0,007 & - & - & - \\
\hline Indicador de Bens & 0,8 & $0,7-0,8$ & $<0,001$ & 1,3 & $1,1-1,6$ & 0,007 & 0,7 & $0,7-0,8$ & $<0,001$ & 0,9 & $0,5-1,6$ & 0,757 \\
\hline $\begin{array}{l}\text { Moradores por } \\
\text { domicílio }\end{array}$ & 1,0 & $1,0-1,1$ & 0,199 & 1,0 & $0,9-1,1$ & 0,943 & 1,1 & $1,0-1,1$ & 0,024 & 1,1 & $0,8-1,6$ & 0,566 \\
\hline
\end{tabular}


Tabela 4 - Modelo final ajustado pelas características individuais e domiciliares das pessoas que receberam atendimento ambulatorial e internação hospitalar que se dizem satisfeitos em relação à qualidade dos profissionais de saúde. Brasil, 2003 e Pernambuco 2005.

Table 4 - Final model adjusted by individual and household characteristics of individuals who received outpatient care and hospitalization and who said they were satisfied with the quality of healthcare workers. Brazil, 2003 and Pernambuco, 2005.

\begin{tabular}{|c|c|c|c|c|c|c|c|c|c|c|c|c|}
\hline \multirow[t]{3}{*}{ Características Gerais } & \multicolumn{6}{|c|}{ Atendimento ambulatorial } & \multicolumn{6}{|c|}{ Internação hospitalar } \\
\hline & \multicolumn{3}{|c|}{ PMS brasileira } & \multicolumn{3}{|c|}{ PMS_AB de Pernambuco } & \multicolumn{3}{|c|}{ PMS brasileira } & \multicolumn{3}{|c|}{ PMS_AB de Pernambuco } \\
\hline & OR & $95 \% \mathrm{Cl}$ & $\mathrm{p}$-value & OR & $95 \% \mathrm{Cl}$ & $\mathrm{p}$-value & OR & $95 \% \mathrm{Cl}$ & $p$-value & OR & $95 \% \mathrm{Cl}$ & $\mathrm{p}$-value \\
\hline \multicolumn{13}{|l|}{ Sexo } \\
\hline Masculino & 1,0 & - & - & & & & & & & & & \\
\hline Feminino & 1,3 & $1,1-1,6$ & 0,010 & & & & & & & & & \\
\hline \multicolumn{13}{|l|}{ Faixa etária } \\
\hline 40 anos e + & & & & & & & 1,0 & - & - & & & \\
\hline 18 a 39 anos & & & & & & & 0,6 & $0,5-0,8$ & $<0,001$ & & & \\
\hline \multicolumn{13}{|l|}{ Escolaridade } \\
\hline $1^{\circ}$ grau completo e + & 1,0 & - & - & 1,0 & - & - & & & & 1,0 & - & - \\
\hline $1^{\circ} \mathrm{grau}$ incompleto & 0,7 & $0,6-0,9$ & 0,007 & 0,6 & $0,4-0,9$ & 0,023 & & & & 0,2 & $0,1-0,6$ & 0,005 \\
\hline \multicolumn{13}{|l|}{ Forma de Pagamento } \\
\hline Não SUS & 1,0 & - & - & & & & 1,0 & - & - & & & \\
\hline SUS & 0,4 & $0,4-0,6$ & $<0,001$ & & & & 0,5 & $0,4-0,7$ & $<0,001$ & & & \\
\hline \multicolumn{13}{|l|}{$\begin{array}{l}\text { Auto-percepção de } \\
\text { saúde }\end{array}$} \\
\hline Boa & 1,0 & - & - & & & & 1,0 & - & - & & & \\
\hline Ruim & 0,7 & $0,6-0,9$ & 0,002 & & & & 0,6 & $0,5-0,8$ & $<0,001$ & & & \\
\hline \multicolumn{13}{|l|}{$\begin{array}{l}\text { Diagnóstico de } \\
\text { depressão }\end{array}$} \\
\hline Não & 1,0 & - & - & & & & & & & & & \\
\hline Sim & 1,2 & $1,0-1,6$ & 0,087 & & & & & & & & & \\
\hline \multicolumn{13}{|l|}{ Estrato geográfico } \\
\hline Sudeste & 1,0 & - & - & & & & 1,0 & - & - & & & \\
\hline Norte & 1,6 & $0,9-2,9$ & 0,099 & & & & 1,0 & $0,6-1,9$ & 0,894 & & & \\
\hline $\begin{array}{l}\text { Nordeste sem } \\
\text { Pernambuco }\end{array}$ & 1,0 & $0,8-1,3$ & 0,842 & & & & 1,2 & $0,8-1,7$ & 0,321 & & & \\
\hline Sul & 1,6 & $1,2-2,2$ & 0,001 & & & & 1,6 & $1,1-2,2$ & 0,006 & & & \\
\hline Centro-Oeste & 1,2 & $0,8-1,8$ & 0,293 & & & & 1,5 & $0,9-2,4$ & 0,144 & & & \\
\hline Pernambuco & 1,3 & $0,8-2,1$ & 0,242 & & & & 0,6 & $0,4-1,1$ & 0,118 & & & \\
\hline Indicador de Bens & 1,2 & $1,1-1,3$ & 0,001 & & & & & & & & & \\
\hline $\begin{array}{l}\text { Moradores por } \\
\text { domicílio }\end{array}$ & 0,9 & $0,9-1,0$ & 0,022 & & & & & & & & & \\
\hline
\end{tabular}

foi a escolaridade. A mesma teve comportamento similar à encontrada na pesquisa nacional (Tabela 4).

A análise das variáveis para as pessoas que relataram internação na PMS brasileira revelou que ser jovem, usuário do SUS e ter saúde percebida como ruim, configura situação de maior insatisfação com o sistema de saúde brasileiro na hora de uma internação.

Os usuários do sistema de saúde da região Sul do país apresentaram maior satisfação em relação à qualidade dos profissionais de saúde, tanto para o atendimento ambulatorial, quanto para as pessoas que se internaram. A análise dos dados das demais regiões do país não revelou associação significativa com a satisfação para os dois tipos de atendimento em relação à qualidade dos profissionais de saúde (Tabela 4).

Quanto à análise das variáveis associadas à satisfação levando-se em conta a avaliação da qualidade dos serviços de saúde, ser usuário do SUS é o fator que mais está associado à insatisfação, tanto para a PMS brasileira, quanto para a PMS-AB realizada em Pernambuco (Tabela 5).

Ainda na Tabela 5, apesar de não ter resultado significante (no nível de 5\%) do ponto de vista estatístico, ser morador do Estado de Pernambuco e da Região Nordeste implicou 
Tabela 5- Modelo final ajustado pelas características individuais e domiciliares das pessoas que receberam atendimento ambulatorial e internação hospitalar que se dizem satisfeitos em relação à qualidade dos serviços de saúde. Brasil, 2003 e Pernambuco, 2005.

Table 5 - Final model adjusted by individual and household characteristics of individuals who received outpatient care and hospitalization and who said they were satisfied with the quality of health services. Brazil, 2003 and Pernambuco, 2005.

\begin{tabular}{|c|c|c|c|c|c|c|c|c|c|c|c|c|}
\hline \multirow[t]{3}{*}{ Características Gerais } & \multicolumn{6}{|c|}{ Atendimento ambulatorial } & \multicolumn{6}{|c|}{ Internação hospitalar } \\
\hline & \multicolumn{3}{|c|}{ PMS brasileira } & \multicolumn{3}{|c|}{ PMS_AB de Pernambuco } & \multicolumn{3}{|c|}{ PMS brasileira } & \multicolumn{3}{|c|}{ PMS_AB de Pernambuco } \\
\hline & OR & $95 \% \mathrm{Cl}$ & $\mathrm{p}$-value & OR & $95 \% \mathrm{Cl}$ & $\mathrm{p}$-value & $\mathrm{OR}$ & $95 \% \mathrm{Cl}$ & $\mathrm{p}$-value & OR & $95 \% \mathrm{Cl}$ & $\mathrm{p}$-value \\
\hline \multicolumn{13}{|l|}{ Escolaridade } \\
\hline $1^{\circ}$ grau completo e + & 1,0 & - & - & & & & & & & & & \\
\hline $1^{\circ} \mathrm{grau}$ incompleto & 1,3 & $1,0-1,6$ & 0,035 & & & & & & & & & \\
\hline \multicolumn{13}{|l|}{ Estado conjugal } \\
\hline Solteiro (a) & & & & & & & 1,0 & - & - & & & \\
\hline Casado (a) & & & & & & & 1,6 & $1,1-2,1$ & 0,004 & & & \\
\hline Separado (a) & & & & & & & 1,0 & $0,6-1,8$ & 0,944 & & & \\
\hline Viúvo (a) & & & & & & & 1,8 & $1,0-3,0$ & 0,035 & & & \\
\hline \multicolumn{13}{|l|}{ Forma de Pagamento } \\
\hline Não SUS & 1,0 & - & - & 1,0 & - & & 1,0 & - & - & & & \\
\hline SUS & 0,3 & $0,2-0,4$ & $<0,001$ & 0,2 & $0,1-0,3$ & $<0,001$ & 0,4 & $0,3-0,5$ & $<0,001$ & & & \\
\hline \multicolumn{13}{|l|}{$\begin{array}{l}\text { Auto-percepção de } \\
\text { saúde }\end{array}$} \\
\hline Boa & 1,0 & - & - & & & & 1,0 & - & - & & & \\
\hline Ruim & 0,8 & $0,6-1,0$ & 0,025 & & & & 0,7 & $0,6-0,9$ & 0,010 & & & \\
\hline \multicolumn{13}{|c|}{$\begin{array}{l}\text { Tristeza nos últimos } 12 \\
\text { meses }\end{array}$} \\
\hline Não & & & & & & & & & & 1,0 & - & \\
\hline$\underline{\operatorname{Sim}}$ & & & & & & & & & & 0,3 & $0,1-0,9$ & 0,032 \\
\hline \multicolumn{13}{|l|}{ Estrato geográfico } \\
\hline Sudeste & 1,0 & - & - & & & & 1,0 & - & - & & & \\
\hline Norte & 1,1 & $0,7-2,0$ & 0,636 & & & & 1,2 & $0,7-2,1$ & 0,589 & & & \\
\hline $\begin{array}{l}\text { Nordeste sem } \\
\text { Pernambuco }\end{array}$ & 0,8 & $0,7-1,1$ & 0,177 & & & & 1,3 & $1,0-1,9$ & 0,088 & & & \\
\hline Sul & 1,5 & $1,1-2,0$ & 0,006 & & & & 0,8 & $0,5-1,1$ & 0,103 & & & \\
\hline Centro-Oeste & 1,1 & $0,7-1,6$ & 0,794 & & & & 0,7 & $0,4-1,2$ & 0,177 & & & \\
\hline Pernambuco & 0,7 & $0,4-1,1$ & 0,098 & & & & 0,6 & $0,3-1,1$ & 0,088 & & & \\
\hline Indicador de Bens & & & & & & & 1,34 & $1,1-1,4$ & $<0,001$ & & & \\
\hline $\begin{array}{l}\text { Moradores por } \\
\text { domicílio }\end{array}$ & 1,0 & $0,9-1,0$ & 0,074 & & & & & & & & & \\
\hline
\end{tabular}

em menor satisfação dos usuários em relação à qualidade dos serviços de saúde. Na análise da qualidade dos profissionais, a Região Sul mais uma vez obteve os maiores graus de satisfação com o atendimento ambulatorial na PMS brasileira.

Em se tratando dos usuários que tiveram alguma internação e que avaliaram a qualidade dos serviços de saúde (hospitais), ser usuário do SUS, ter autopercepção de saúde ruim e ser morador de Pernambuco revelaram os piores graus de satisfação nesta análise (Tabela 5).

O modelo final ajustado para a análise do atendimento ambulatorial quanto à resolutividade dos problemas de saúde revelou que, neste quesito, as mulheres se mostraram mais insatisfeitas com o resultado esperado do atendimento em saúde. Na PMS brasileira, de forma aparentemente incongruente, os resultados encontrados revelaram que ter baixa escolaridade, ser usuário do SUS e ser usuário do Sistema de Saúde de Pernambuco geraram maiores chances de satisfação (Tabela 6).

Quando o atendimento do usuário se deu em nível ambulatorial no Estado de Pernambuco (PMS-AB), observou-se uma situação inversa àquela encontrada na pesquisa nacional, ou seja, ter baixa esco- 
Tabela 6- Modelo final ajustado pelas características individuais e domiciliares das pessoas que receberam atendimento ambulatorial e internação hospitalar que se dizem satisfeitos em relação à resolutividade dos serviços de saúde. Brasil, 2003 e Pernambuco, 2005.

Table 6 - Final model adjusted by individual and household characteristics of individuals who received outpatient care and hospitalization and who said they were satisfied with the level of resolution of healthcare services. Brazil, 2003 and Pernambuco, 2005.

\begin{tabular}{|c|c|c|c|c|c|c|c|c|c|c|c|c|}
\hline \multirow[t]{3}{*}{ Características Gerais } & \multicolumn{6}{|c|}{ Atendimento ambulatorial } & \multicolumn{6}{|c|}{ Internação hospitalar } \\
\hline & \multicolumn{3}{|c|}{ PMS brasileira } & \multicolumn{3}{|c|}{ PMS_AB de Pernambuco } & \multicolumn{3}{|c|}{ PMS brasileira } & \multicolumn{3}{|c|}{ PMS_AB de Pernambuco } \\
\hline & OR & $95 \% \mathrm{Cl}$ & $p$-value & $\mathrm{OR}$ & $95 \% \mathrm{Cl}$ & $p$-value & OR & $95 \% \mathrm{Cl}$ & $\mathrm{p}$-value & $\mathrm{OR}$ & $95 \% \mathrm{Cl}$ & $\mathrm{p}$-value \\
\hline \multicolumn{13}{|l|}{ Sexo } \\
\hline Masculino & 1,0 & - & - & & & & & & & & & \\
\hline Feminino & 0,8 & $0,6-0,9$ & 0,005 & & & & & & & & & \\
\hline \multicolumn{13}{|l|}{ Faixa etária } \\
\hline 40 anos e + & & & & & & & & & & 1,0 & - & - \\
\hline 18 a 39 anos & & & & & & & & & & 0,2 & $0,1-0,7$ & 0,010 \\
\hline \multicolumn{13}{|l|}{ Escolaridade } \\
\hline $1^{\circ}$ grau completo e + & 1,0 & - & - & 1,0 & - & - & & & & & & \\
\hline $1^{\circ} \mathrm{grau}$ incompleto & 1,4 & $1,1-1,7$ & 0,002 & 0,4 & $0,2-0,6$ & $<0,001$ & & & & & & \\
\hline \multicolumn{13}{|l|}{ Forma de Pagamento } \\
\hline Não SUS & 1,0 & - & - & 1,0 & - & - & & & & & & \\
\hline SUS & 1,2 & $1,0-1,5$ & 0,056 & 0,7 & $0,4-1,0$ & 0,049 & & & & & & \\
\hline \multicolumn{13}{|l|}{$\begin{array}{l}\text { Auto-percepção de } \\
\text { saúde }\end{array}$} \\
\hline Boa & & & & & & & 1,0 & - & - & & & \\
\hline Ruim & & & & & & & 1,3 & $1,0-1,6$ & 0,029 & & & \\
\hline \multicolumn{13}{|l|}{ Tipo de construção } \\
\hline Material permanente & & & & 1,0 & - & - & & & & & & \\
\hline Material não permanente & & & & 0,1 & $0,0-0,8$ & 0,025 & & & & & & \\
\hline \multicolumn{13}{|l|}{ Estrato geográfico } \\
\hline Sudeste & 1,0 & - & - & & & & 1,0 & - & - & & & \\
\hline Norte & 0,3 & $0,2-0,6$ & $<0,001$ & & & & 0,8 & $0,5-1,5$ & 0,531 & & & \\
\hline $\begin{array}{l}\text { Nordeste sem } \\
\text { Pernambuco }\end{array}$ & 1,1 & $0,9-1,4$ & 0,373 & & & & 0,8 & $0,6-1,1$ & 0,168 & & & \\
\hline Sul & 0,8 & $0,6-1,0$ & 0,047 & & & & 0,9 & $0,7-1,3$ & 0,649 & & & \\
\hline Centro-Oeste & 1,1 & $0,7-1,6$ & 0,640 & & & & 0,7 & $0,4-1,1$ & 0,138 & & & \\
\hline Pernambuco & 1,2 & $0,8-1,9$ & 0,381 & & & & 0,3 & $0,2-0,6$ & $<0,001$ & & & \\
\hline Indicador de Bens & 0,9 & $0,8-1,0$ & 0,006 & & & & 0,7 & $0,6-0,8$ & $<0,001$ & & & \\
\hline $\begin{array}{l}\text { Moradores por } \\
\text { domicílio }\end{array}$ & & & & & & & 1,1 & $1,0-1,1$ & 0,078 & & & \\
\hline
\end{tabular}

laridade e ser usuário do SUS no Estado de Pernambuco deixa os indivíduos mais insatisfeitos com o sistema de saúde na hora da resolução dos problemas de saúde (Tabela 6). No caso de haver uma internação, a análise dos dados desta pesquisa revelou que ser jovem é a única variável que se mostrou significativamente associada à insatisfação com a resolução dos problemas de saúde no estado de Pernambuco.

Para o Brasil como um todo, a análise da resolutividade dos problemas de saúde quando o sujeito precisou ser internado revelou que, independentemente da região de moradia, toda a população tem mais chance de se sentir insatisfeita neste quesito, porém estes resultados não foram estatisticamente significantes ao nível de 5\%, com exceção do Estado de Pernambuco, que revelou de forma significativa uma maior chance de insatisfação neste quesito.

\section{Discussão}

Dados do Banco Central do Brasil ${ }^{12}$ revelaram que as diferenças encontradas no IDH das diversas regiões geográficas do país são acentuadas, variando de 0,720 na 
Região Nordeste a 0,829 na Região Sul. Essas diferenças acabam refletindo na qualidade de vida e na saúde dessas populações. Para a Comissão Nacional Sobre Determinantes Sociais da Saúde ${ }^{18}$, as condições socioeconômicas, culturais e ambientais de uma sociedade acabam gerando uma estratificação socioeconômica dos indivíduos e grupos populacionais, conferindo-lhes posições sociais distintas que acabam provocando diferenciais de saúde e de percepção.

De um modo geral, o fato de se ter encontrado maiores chances de satisfação por parte dos usuários da Região Sul, enquanto na Região Nordeste, particularmente no Estado de Pernambuco, essas chances são as menores encontradas no país, faz com que esses achados reforcem a idéia de que as desigualdades entre as diversas regiões, ainda persistam no Brasil, corroborando os achados de Lima-Costa et al. ${ }^{14}$ quanto à análise das PNAD de 1998 e 2003.

Segundo Szwarcwald et al. ${ }^{19}$, no Brasil, $58 \%$ da população se mostra insatisfeita com o funcionamento da assistência de saúde. Alguns fatores influenciam mais este grau de insatisfação, como foi demonstrado por alguns autores ${ }^{17,20-22}$, em que o acesso e a qualidade dos serviços foram os piores indicadores identificados pelos usuários.

Os achados encontrados neste estudo corroboram com os autores citados acima, pois evidenciam que, na análise da PMS brasileira, o fato de residir em Pernambuco estava mais associado à insatisfação quanto à qualidade dos serviços de saúde, qualidade esta influenciada também pela questão do acesso aos serviços, independentemente do tipo de assistência recebida, bem como em relação à resolução dos problemas durante a internação.

Uma limitação encontrada neste estudo foi o tamanho da amostra da PMS-AB realizada em Pernambuco, que foi considerada pequena para as análises estatísticas utilizadas, dificultando, por exemplo, a associação da maioria das variáveis independentes com a satisfação quando da análise dos resultados do modelo final da regressão logística.

Para Travassos, Oliveira eViacava ${ }^{23}$, entre os anos de 1998 e 2003 houve um aumento das desigualdades geográficas no acesso aos serviços de saúde, influenciado pela melhora de acesso nas regiões mais desenvolvidas em comparação com as menos desenvolvidas (resultados da PNAD). Ainda segundo esses autores, na análise desagregada por regiões persistiu o padrão de desigualdades influenciadas particularmente pela renda $\mathrm{e}$ escolaridade.

Messias $^{24}$, que analisou os dados do censo de 2000, coloca que o PIB per capita, a distribuição de renda e a taxa de analfabetismo estão associados à expectativa de vida; portanto, renda e escolaridade estão fortemente associados a resultados de saúde. Neste estudo, a escolaridade foi uma importante variável que gerou maior insatisfação quando da análise da qualidade dos profissionais de saúde, tanto na pesquisa nacional quanto na pesquisa local, independentemente do tipo de assistência recebida.

A Comissão Nacional Sobre Determinantes Sociais da Saúde ${ }^{18}$ coloca que os efeitos do nível de instrução aparecem na forma de percepção dos problemas de saúde; na capacidade de entendimento das informações sobre saúde; na adoção de estilos de vida saudáveis; no consumo e na utilização dos serviços de saúde bem como na adesão aos procedimentos terapêuticos. Aqui cabe acrescentar que os resultados encontrados revelaram que, além desses fatores acima citados, o nível de instrução tem efeito direto na satisfação com os serviços de saúde percebidos pelos usuários. Para Weiss ${ }^{25}$, que fez uma extensa revisão sobre a satisfação dos pacientes, as características dos profissionais de saúde que prestam o atendimento, são fundamentais na determinação da satisfação dos usuários.

Para Trad et al ${ }^{5}$, os usuários do Programa de Saúde da Família expressam um alto grau de satisfação em relação à dimensão relacional, em todos os seus atributos: respeito, consideração, escuta, compreensão, acolhida e gentileza por parte dos profissionais da equipe; portanto, em relação à qualidade dos profissionais de saúde. 
Neste quesito, os níveis de satisfação encontrados neste estudo não corroboram com os achados da pesquisa citada anteriormente, pois as análises revelaram desigualdades mais evidentes quando se abordou a qualidade dos profissionais de saúde, quesito em que as pessoas de baixa escolaridade apresentaram propensão à insatisfação. Nossos resultados corroboram com os achados de Dachs ${ }^{7}$ e Marins et al. ${ }^{26}$, onde as desigualdades de saúde foram mais evidentes entre as pessoas que tinham baixa escolaridade.

Para Ribeiro et al. ${ }^{27}$, Gouveia et al. ${ }^{17,28}$, que analisaram o perfil de indivíduos que procuraram os serviços de saúde no país, foi observado que existe predominância de usuários do SUS e de baixa escolaridade, o que corrobora com os achados deste trabalho.

Neste estudo, além do perfil dos indivíduos ser semelhante ao encontrado nas pesquisas referidas acima, observou-se que as chances de uma maior insatisfação com a qualidade dos profissionais de saúde recaiu significativamente sobre os indivíduos de baixa escolaridade (variável proxi do nível socioeconômico) no país como um todo, e de forma particular no Estado de Pernambuco. Na PMS-AB de Pernambuco, a baixa escolaridade significa uma maior chance de insatisfação, enquanto, para a PMS brasileira, além da baixa escolaridade, ser usuário do SUS refletiu menores chances de satisfação.

O processo de amostragem da PMS nacional não permitiu que os resultados fossem avaliados por estados da federação, e por isso foi necessário realizar uma pesquisa posterior em alguns Estados do país que proporcionassem esta avaliação e possibilitassem algumas comparações. As duas pesquisas (PMS e PMS-AB) foram voltadas para avaliação do desempenho do sistema de saúde, mas a pesquisa de âmbito estadual foi mais aprofundada em algumas questões, como, por exemplo, a possibilidade de avaliação da Estratégia do Programa Saúde da Família. O módulo do instrumento de coleta referente à avaliação da satisfação não foi diferente nas duas pesquisas, o que permitiu estabelecer comparações dos graus de satisfação em dois momentos distintos.

Apesar de não estarem tão fortemente assinaladas, as diferenças regionais na satisfação com o atendimento de saúde no Brasil persistem, sugerindo que somente com a aplicação real do conceito de equidade (os recursos do sistema de saúde devem ser distribuídos de forma atenta às desigualdades existentes, com o objetivo de ajustar as suas ações às necessidades de cada parcela da população), que constitui um dos princípios do SUS, será possível reduzir essas diferenças. Existem formas de diminuir essas desigualdades mediante ações efetivas na prática do atendimento, com a melhoria do acesso, um maior reconhecimento dos profissionais de saúde, e a percepção de que é possível uma melhoria na qualidade do atendimento, mesmo sob um contexto de enorme e reconhecida disparidade socioeconômica no país.

Sintetizando, para o usuário brasileiro, ser jovem, usuário exclusivo do SUS, ter baixa escolaridade e autopercepção de saúde ruim gerou mais chances de insatisfação dos usuários com relação ao sistema de saúde brasileiro. De modo geral, os indivíduos residentes na Região Sul do país apresentam maiores chances de se sentirem satisfeitos com o atendimento em saúde, enquanto para o Nordeste, e em particular para Pernambuco, as chances de satisfação são menores quando se avalia a qualidade dos serviços de saúde. Os moradores desse Estado apresentaram maior insatisfação na resolução de seus problemas de saúde, quando precisaram de internação. 


\section{Referências}

1. Paim JS. Avaliação em saúde: uma prática em construção no Brasil. In: Hartz ZMA, Vieira da Silva LM (orgs.). Avaliação em saúde. Dos modelos teóricos à prática na avaliação de programas e sistemas de saúde. Salvador: EDUFBA; Rio de Janeiro: FIOCRUZ; 2005: 9-10.

2. Donabedian A. The definition of quality: a conceptual exploration. Explorations is quality assessment and monitoring. V1: The definition of quality and approaches to its assessment. Ann Arbor, Michigan: Health Administration Press; 1888. p. 3-31.

3. Esperidião M, Trad LAB. - Avaliação de satisfação de usuários. Ciênc Saúde Coletiva 2005; (S10); 303-12.

4. Donabedian A. La calidad de la atención médicadefinición y métodos de evaluatión. México, DF: La Prensa Mexicana; 1984.

5. Trad, LAB, Bastos ACS. O impacto sócio-cultural do Programa de saúde da Família (PSF): uma proposta de avaliação. Cad Saúde Pública 1988; 14(2): 429-35.

6. Murray CJL, Kawabata K, Valentine N. People's Experience Versus People's Expectations. Satisfaction measures are profoundly influenced by expectations, say these WHO researchers. Health Affairs 2001; 20(3): 21-4.

7. Dachs, JNW. Determinantes das desigualdades na auto-avaliação do estado de saúde no Brasil: análise dos dados da PNAD/1998. Ciênc Saúde Coletiva 2002; 7(4): 641-57.

8. Organização Pan-Americana da Saúde (OPAS). Saúde nas Américas. Washington: Opas; 2007. v. 1. (Publicação Científica e Técnica, 622).

9. Mendes EV. Os grandes dilemas do SUS: tomo I. Salvador, BA: Casa da Qualidade Editora; 2001.

10. Szwarcwald CL, Bastos FI, Andrade CLT. Medidas de desigualdad em salud: la discusión de alguns aspectos metodológicos com uma aplicación para la mortalidad neonatal em el Município de Rio de Janeiro, 2000. Cad Saúde Pública 2002; 18(4): 959-70.

11. Organização Pan-Americana da Saúde (OPAS). La Salud en las Américas, vol. 1 Washington: OPAS; 1998.

12. Banco Central do Brasil. Evolução do IDH das Grandes Regiões e Unidades da Federação. Boletim Regional do Banco Central do Brasil. Disponível em http:// www.bcb.gov.br /pec/boletimregional/port/2009/01/ br200901b1p.pdf [Acessado em 20 de fevereiro de 2009].

13. NEGRI, B. - A política de Saúde no Brasil nos anos 90: Avanços e Limites. Ministério da Saúde; Brasília: Ministério da Saúde, 2002.
14. Lima-Costa MF, Matos DL, Camarano AA. Evolução das desigualdades sociais em saúde entre idosos e adultos brasileiros: um estudo baseado na Pesquisa Nacional por Amostra de Domicílios (PNAD 1998, 2003). Ciênc Saúde Coletiva 2006; 11(4): 941-50.

15. Vasconcellos MTL, Silva PLN, Szwarcwald CL. Sampling design for the World Health Survey in Brazil. Cad Saúde Pública 2005; 21(S1): S89-S99.

16. Szwarcwald CL, Leal MC, Gouveia GC, Souza WV. Desigualdades socioeconômicas em saúde no Brasil: resultados da Pesquisa Mundial de Saúde, 2003. Rev Bras Saúde Matern Infant 2005; 5(S1): S11-S22.

17. Gouveia GC, Souza WV, Luna CF, Souza-Júnior PRB, Szwarcwald CL. Health care users' satisfaction in Brazil, 2003. Cad Saúde Pública 2005; 21(S1): S109-S18.

18. Comissão Nacional sobre Determinantes Sociais da Saúde. As causas sociais das iniqüidades em saúde no Brasil. Rio de Janeiro: Editora FIOCRUZ; 2008.

19. Szwarcwald CL, Viacava F, Vasconcellos MTL, Leal MC, Azevedo LO, Queiroz RSB, Carvalho MF, Souza Júnior PRB, Gama SGN, Fonseca MG. Pesquisa Mundial de Saúde - 2003: O Brasil em números. RADIS 2004; 1(23): 14-33.

20. Lemme AC, Noronha G, Resende JB. A Satisfação do Usuário em Hospital Universitário. Rev Saúde Pública 1991; 25(1): 41-6.

21. Brasil. Ministério da Saúde. Conselho Nacional de Secretários de Saúde (CONASS). A Saúde na Opinião dos Brasileiros. Brasília: CONASS; 2003. Disponível em http://bvsms.saude.gov.br/bvs/publicacoes/opiniao_ brasileiros1.pdf [Acessado em 12 de fevereiro de 2009].

22. Assis MMA, Villa TCS, Nascimento MAA. Acesso aos serviços de saúde: uma possibilidade a ser construída na prática. Ciênc Saúde Coletiva 2003; 8(3): 815-23.

23. Travassos C, Oliveira EXG, Viacava F. Desigualdades geográficas e sociais no acesso aos serviços de saúde no Brasil: 1998 e 2003. Ciên Saúde Coletiva 2006; 11(4): 97586.

24. Messias E. Income inequality, illiteracy rate, and life expectancy in Brazil. Am J Public Health 2003; 93(8): 1294-6.

25. Weiss LG. Patient satisfaction with primary medical care: evaluation of sociodemographic and predispositional factors. Med Care 1998; 26: 383-92.

26. Marins VMR et al. The association between socioeconomic indicators and cardiovascular disease risk factors in Rio de Janeiro, Brazil. J Biosoc Sci 2007; 39: 221-9. 
27. Ribeiro MCSA et al. Perfil sociodemigráfico e padrão de utilização de serviços de saúde para usuários e nãousuários do SUS - Pnad 2003. Ciênc Saúde Coletiva 2006; 11(4): 1011-22.
28. Gouveia GC, Souza WV, Luna CF, Souza-Júnior PRB, Szwarcwald CL. Satisfação dos usuários com a assistência de saúde no Estado de Pernambuco - Brasil, 2005. Ciênc Saúde Coletiva, aprovado em 16 de fevereiro de 2009. No prelo.

Recebido em: 08/04/09

Versão final reapresentada em: 02/06/09

Aprovado em: 23/06/09 